INPLASY

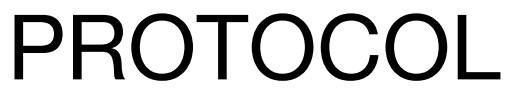

To cite: Cao et al. The Efficacy and Safety of Oral Chinese Herbal Medicine for Depression: A Protocol for Systematic Review and Network Meta-analysis. Inplasy protocol 2020120084. doi:

10.37766/inplasy2020.12.0084

Received: 16 December 2020

Published: 16 December 2020

Corresponding author:

Yue Cao

942133430@qq.com

Author Affiliation:

Chengdu University of TCM

Support: SQ2018YFC170240.

Review Stage at time of this submission: Preliminary

searches.

Conflicts of interest:

None.

\section{The Efficacy and Safety of Oral Chinese Herbal Medicine for Depression: A Protocol for Systematic Review and Network Meta-analysis}

Cao, Y1; Yuan, J2; Cao, W3; Wen, CB4.

Review question / Objective: P: Participants were people diagnosed with depression through a systematical clinical interview or a validated depression scoring system. The author have no conflicts of interest to disclose. I: We will include randomized controlled trials to evaluate the efficacy of oral Chinese herbal medicine in the treatment of depression. All oral Chinese herbal medicines suitable for depression will participate in our research. There is no limit to the method of administration and duration of treatment. Studies that combine drug therapy with other treatments or two drug treatments will be excluded. C: The control group will include Conventional antidepressants, routine nursing and blank control group, which will be regarded as a single node in the network meta-analysis. 0 : The main outcome is the change in the depression score from baseline to endpoint, measured by validated depression scoring systems, Hamilton Depression Rating Scale (HAMD), In order to understand safety of them, We will include adverse reaction parameters. In order to examine the possible maintenance effects of oral Chinese herbal medicine treatments, effect at the end of follow-up will also be evaluated as the secondary outcome. S: RCTs.

INPLASY registration number: This protocol was registered with the International Platform of Registered Systematic Review and Meta-Analysis Protocols (INPLASY) on 16 December 2020 and was last updated on 16 December 2020 (registration number INPLASY2020120084).

\section{INTRODUCTION}

Review question / Objective: P: Participants were people diagnosed with depression through a systematical clinical interview or a validated depression scoring system. The author have no conflicts of interest to disclose. I: We will include randomized controlled trials to evaluate the efficacy of oral Chinese herbal medicine in 
the treatment of depression. All oral Chinese herbal medicines suitable for depression will participate in our research.There is no limit to the method of administration and duration of treatment. Studies that combine drug therapy with other treatments or two drug treatments will be excluded. C: The control group will include Conventional antidepressants, routine nursing and blank control group, which will be regarded as a single node in the network meta-analysis. O: The main outcome is the change in the depression score from baseline to endpoint, measured by validated depression scoring systems, Hamilton Depression Rating Scale (HAMD), In order to understand safety of them, We will include adverse reaction parameters. In order to examine the possible maintenance effects of oral Chinese herbal medicine treatments, effect at the end of follow-up will also be evaluated as the secondary outcome. S: RCTs.

Condition being studied: At present, most of the randomized controlled trials(RCTs) of OCHM in the treatment of depression and traditional Meta analysis generally choose positive drugs or blank group as the control group. The results show that OCHM can improve the effectiveness index, improve patients' anxiety and depression symptoms and is safer, but there is a lack of comparison between different traditional Chinese medicine treatment methods. In order to meet the needs of comparing the efficacy and safety of these prescriptions, the biggest advantage of (NMA), NMA in Bayesian mesh Meta analysis is that based on the common comparison group, we can associate two interventions that lack direct comparative evidence, use indirect evidence to estimate their relative effects and rank them, and then choose the best treatment scheme. NMA breaks through the limitation that traditional Meta analysis can only compare the two intervention measures, and is more consistent with the complex characteristics of "different treatment of the same disease" of traditional Chinese medicine, and provides evidence-based medicine for clinical application.

\section{METHODS}

Participant or population: Participants were people diagnosed with depression through a systematical clinical interview or a validated depression scoring system.

Intervention: We will include randomized controlled trials to evaluate the efficacy of oral Chinese herbal medicine in the treatment of depression. All oral Chinese herbal medicines suitable for depression will participate in our research.There is no limit to the method of administration and duration of treatment. Studies that combine drug therapy with other treatments or two drug treatments will be excluded.

Comparator: The control group will include Conventional antidepressants, routine nursing and blank control group, which will be regarded as a single node in the network meta-analysis.

Study designs to be included: Randomized controlled trials (RCTs).

Eligibility criteria: We will exclude the researches with quasi-random allocation or the studies without available data.Duplicate publication will also be excluded in the current study, In order to ensure the quality of the literature, the literature that does not belong to the source journals of the Overview of Chinese Core Journals will not be included in this study.

Information sources: Electronic bibliographic databases including EMBASE, PubMed, Scopus, The Cochrane Library, Web of Science, China National Knowledge Infrastructure (CNKI), VIP Database, Wanfang Database and Chinese Biomedical Literature Database will be searched for relevant randomized controlled trials (RCTs) of Chinese herbal medicine for depression.

Main outcome(s): The main outcome is the change in the depression score from baseline to endpoint, measured by validated depression scoring systems, 
Hamilton Depression Rating Scale (HAMD), In order to understand safety of them, We will include adverse reaction parameters.

Quality assessment / Risk of bias analysis: Two independent reviewers (JY and WC) will assess the risk of bias of included studies according to the Cochrane Handbook, which includes random sequence generation, allocation concealment, blinding of participants, personnel and outcome assessors, incomplete outcome data, selective reporting, and other sources of bias.We will classify each domain into 3 levels: high, unclear or low risk of bias. Any variation in opinion between reviewers will be resolved by discussion or consultation with a third reviewer (CBW).

Strategy of data synthesis: We will conduct the conventional pairwise meta-analysis for direct comparisons. The effect size of continuous variable data will be calculated with standardized mean difference (SMD), while the effect size of categorical variable data with risk ratio (RR). The related $95 \%$ confidence intervals (Cls) of SMD and RR will also be computed. For indirect comparisons, a network meta-analysis is required to mix results to improve statistical efficacy. The network meta analysis will be performed with the "netmeta"package of $R$ software, which based on frequentist method.The direct and indirect comparison results will be presented as a network diagram.Before the combination of effect size, the homogeneity will be analyzed by 12 statistic to check whether the results of individual studies are mergeable. When $12 \leq 50 \%$, the fixed effect model will be used to combine the effect size. Conversely, the random effect model will be used for meta-analysis after excluding the influence of apparent clinical heterogeneity.

Subgroup analysis: The subgroup analysis will be conducted to resolve the potential high heterogeneity across RCTs.

Sensibility analysis: We will conduct sensitivity analysis to verify the robustness of the results. The research with unclear or high risk of bias will be exclude to check if the results would change.

Language: The written language is limited in English or Chinese.

Country(ies) involved: China.

Keywords: network meta-analysis, protocol, oral Chinese herbal medicine, depression.

Contributions of each author:

Author 1 - Yue Cao.

Author 2 - Jie Yuan.

Author 3 - Wei Cao.

Author 4 - ChuanBiao Wen. 